\title{
Inelastic carrier lifetime in graphene
}

\author{
E. H. Hwang, ${ }^{1}$ Ben Yu-Kuang Hu, ${ }^{2,1}$ and S. Das Sarma ${ }^{1}$ \\ ${ }^{1}$ Condensed Matter Theory Center, Department of Physics, \\ University of Maryland, College Park, Maryland 20742-4111 and \\ ${ }^{2}$ Department of Physics, University of Akron, Akron, OH 44325-4001
}

(Dated: February 6, 2008)

\begin{abstract}
We consider hot carrier inelastic scattering due to electron-electron interactions in graphene, as functions of carrier energy and density. We calculate the imaginary part of the zero-temperature quasiparticle self-energy for doped graphene, utlizing the $G_{0} W$ and random phases approximations. Using the full dynamically screened Coulomb interaction, we obtain the inelastic quasiparticle lifetimes and associated mean free paths. The linear dispersion of graphene gives lifetime energy dependences that are qualitatively different from those of parabolic-band semiconductors. We also get good agreement with data from angle-resolved photoemission spectroscopy experiments.
\end{abstract}

PACS numbers: 81.05.Uw; 71.10.-w; 71.18.+y; 73.63.Bd

\section{INTRODUCTION}

Graphene, a single layer of carbon atoms covalently bonded together in a honeycomb structure (as in a monolayer of graphite), was previouly thought to be unstable and hence non-existent in a free state. Recently, however, Novoselov et al. reported $\underline{1}$ that they had succeeded in fabricating single graphene sheets. Subsequently, several experimental groups have reported interesting transport and spectroscopic measurements $2,3,4$, which has led to experimental and theoretical interest in this field that is rapidly burgeoning.

The overlap of the $\pi_{z}$ orbitals between neighboring carbon atoms in the graphene plane is accurately described by a tight-binding Hamiltonian. Around the $K$ and $K^{\prime}$ points (often called Dirac points) which are at the corners of the hexagonal Brillouin zone, the kinetic energy term of the Hamiltonian is well-approximated by a twodimensional (2D) Dirac equation for massless particles, $\hat{H}_{0}=-v_{0}\left(\sigma_{x} \hat{k}_{x}+\sigma_{y} \hat{k}_{y}\right)$, where $\sigma_{x}$ and $\sigma_{y}$ are $2 \times 2$ Pauli spinors and $\boldsymbol{k}$ is the momentum relative to the Dirac points ( $\hbar=1$ throughout this paper). The two components of the spinors correspond to occupancy of the two sublattices of the honeycomb structure in a hexagonal lattice. This $\hat{H}_{0}$ gives a linear energy dispersion relation $\epsilon_{\boldsymbol{k}, s}=s v_{0}|\boldsymbol{k}|$, where $s=+1(-1)$ for the conduction (valence) band. The corresponding density of states (DOS) is $D(\epsilon)=g_{s} g_{v}|\epsilon| /\left(2 \pi v_{0}^{2}\right)$, where $g_{s}=2, g_{v}=2$ are the spin and valley (i.e., $K$ and $K^{\prime}$ points) degeneracies, respectively. Thus, graphene is a gapless semiconductor. In intrinsic graphene, the Fermi level lies at the Dirac points, but as with other semiconductors it is possible to shift the Fermi level either by doping the sample or applying an external gate voltage, which introduces $2 \mathrm{D}$ free carriers (electrons or holes) producing extrinsic graphene with gate voltage induced tunable carrier density. The Fermi momentum $\left(k_{F}\right)$ and the Fermi energy $\left(E_{F}\right.$, relative to the Dirac point energy) of graphene are given by $k_{F}=\left(4 \pi n / g_{s} g_{v}\right)^{1 / 2}$ and $\left|E_{F}\right|=v_{0} k_{F}$ where $n$ is the $2 \mathrm{D}$ carrier (electron or hole) density.

Interparticle interactions can significantly affect elec- tronic properties, particularly in systems of reduced dimensionality. Moreover, the linear energy dispersion around the Dirac points gives condensed matter experimentalists a unique opportunity to study interaction effects on effectively massless particles. In this paper, we focus on the effect of electron-electron $(e-e)$ interaction effects on the imaginary part of quasiparticle selfenergies, $\operatorname{Im}[\Sigma]$. From $\operatorname{Im}[\Sigma]$, we can extract the quasiparticle lifetime, which gives information that is relevant both to fundamental questions, such as whether or not the system is a Fermi liquid, and to possible applications, such as the energy dissipation rate of injected carriers in a graphene-based device. In particular, an important physical quantity of both fundamental and technological significance is the hot carrier mean free path, which we calculate as a functions of energy, density and in-plane dielectric constant. Furthermore, $\operatorname{Im}[\Sigma]$, being the width of the quasiparticle spectral function, is related to measurements in angle resolved photoemission spectroscopy (ARPES).

The rest of the paper is organized as follows. In section II we discuss the general theory of self-energy of graphene. In section III we present our calculated quasiparticle damping rate of graphene and compare with the damping rate of parabolic $2 \mathrm{D}$ systems. In section IV we show the detail reuslts of the self-energy, and finally we conclude in section $\mathrm{V}$.

\section{THEORY}

We evaluate the self-energy $\Sigma$ within the leading-order ring-diagram $G_{0} W$ approximation, which is appropriate for weak-coupling systems, given by $\underline{\underline{5}}$

$$
\begin{array}{r}
\Sigma_{s}\left(\boldsymbol{k}, i \omega_{n}\right)=-k_{B} T \sum_{s^{\prime}} \sum_{\boldsymbol{q}, i \nu_{n}} G_{0, s^{\prime}}\left(\boldsymbol{k}+\boldsymbol{q}, i \omega_{n}+i \nu_{n}\right) \\
\times W\left(q, i \nu_{n}\right) F_{s s^{\prime}}(\boldsymbol{k}, \boldsymbol{k}+\boldsymbol{q}) .
\end{array}
$$

Here, $T$ is temperature, $s, s^{\prime}= \pm 1$ are band indices, $G_{0}$ is the bare Green's function, $\omega_{n}, \nu_{n}$ are Matsubara 
fermion and boson frequencies, respectively, $W$ is the screened Coulomb interaction, and $F_{s s^{\prime}}\left(\boldsymbol{k}, \boldsymbol{k}^{\prime}\right)=\frac{1}{2}(1+$ $\left.s s^{\prime} \cos \theta_{\boldsymbol{k} \boldsymbol{k}^{\prime}}\right)$, where $\theta_{\boldsymbol{k} \boldsymbol{k}^{\prime}}$ is the angle between $\boldsymbol{k}, \boldsymbol{k}^{\prime}$, arises from the overlap of $|s \boldsymbol{k}\rangle$ and $\left|s^{\prime} \boldsymbol{k}^{\prime}\right\rangle$. The screened interaction $W\left(q, i \nu_{n}\right)=V_{c}(q) / \epsilon\left(q, i \nu_{n}\right)$, where $V_{c}(q)=2 \pi e^{2} / \kappa q$ is the bare Coulomb potential ( $\kappa=$ background dielectric constant), and $\varepsilon\left(q, i \nu_{n}\right)$ is the $2 \mathrm{D}$ dynamical dielectric function. In the random phase approximation $\varepsilon\left(q, i \nu_{n}\right)=$ $1-V_{c}(q) \Pi_{0}\left(q, i \nu_{n}\right)$, where the irreducible polarizability $\Pi_{0}$ is approximated by the bare bubble diagram 6,7 , which gives the familiar Lindhard expression [with a modification to include the form factor $\left.F_{s s^{\prime}}\left(\boldsymbol{k}, \boldsymbol{k}^{\prime}\right)\right]$.

The self-energy approximation described by Eq. (11) should be an excellent approximation for graphene since graphene is inherently a weak-coupling (or "highdensity" in parabolic-band systems) 2D system ${ }^{8}$. The measure of the ratio of the potential to the kinetic energy in graphene is $r_{s}=e^{2} /\left(\kappa v_{0}\right) \approx 2.2 / \kappa$, where $\kappa$ is the effective dielectric constant of the graphene layer and the media surrounding it. (Note that, unlike regular parabolic-band systems, $r_{s}$ in graphene does not depend on density.) Typically in graphene, $r_{s}<1$, implying that the system is in the weak-coupling regime.

After the standard procedure of analytical continuation from $i \omega_{n}$ to $\omega+i 0^{+}$, the retarded self-energy can be separated into the exchange and correlation parts $\Sigma_{s}^{\mathrm{ret}}(\mathbf{k}, \omega)=\Sigma_{s}^{\mathrm{ex}}(\mathbf{k})+\Sigma_{s}^{\mathrm{cor}}(\mathbf{k}, \omega) . \underline{\underline{5}}$ The exchange part is given by

$$
\Sigma_{s}^{\mathrm{ex}}(\mathbf{k})=-\sum_{s^{\prime} \mathbf{q}} n_{F}\left(\xi_{\mathbf{k}+\mathbf{q}, s^{\prime}}\right) V_{c}(\mathbf{q}) F_{s s^{\prime}}(\mathbf{k}, \mathbf{k}+\mathbf{q}),
$$

where $n_{F}$ is the Fermi function, and $\xi_{\boldsymbol{k}, s}=\epsilon_{\boldsymbol{k}, s}-\mu$ is the electron energy relative to the chemical potential $\mu$. This term in graphene is discussed in Ref. 9. At typical experimental densities, the Fermi temperature $T_{F} \equiv\left|E_{F}\right| / k_{B}$ in graphene is very high compared to the temperature of the sample (e.g., for $n=10^{13} \mathrm{~cm}^{-2}, T_{F} \approx 3.1 \times 10^{3} \mathrm{~K}$, and $T_{F} \propto \sqrt{n}$ ). Therefore, it is an excellent approximation to set the temperature $T=0$, which we do in the rest of this paper. This implies that $n_{F}\left(\xi_{\mathbf{k} s}\right)=\theta\left(\xi_{\mathbf{k} s}\right)$ (where $\theta$ is Heaviside unit step function), and $\mu=E_{F}$.

The correlation part, $\Sigma_{s}^{\text {cor }}(\mathbf{k}, \omega)$, is defined to be the part of $\Sigma_{s}^{\text {ret }}(\mathbf{k}, \omega)$ not included in $\Sigma_{s}^{\mathrm{ex}}(\mathbf{k})$. In the $G_{0} W$ approximation, the $\Sigma_{s}^{\text {cor }}(\mathbf{k}, \omega)$ can be decomposed into the line and pole contributions, $\Sigma^{\text {cor }}=\Sigma^{\text {line }}+\Sigma^{\text {pole }}, 10$ where

$$
\begin{gathered}
\Sigma_{s}^{\operatorname{line}}(\mathbf{k}, \omega)=-\sum_{s^{\prime} \mathbf{q}} \int_{-\infty}^{\infty} \frac{d \omega^{\prime}}{2 \pi} \frac{V_{c}(\mathbf{q}) F_{s s^{\prime}}(\mathbf{k}, \mathbf{k}+\mathbf{q})}{\xi_{\mathbf{k}+\mathbf{q}, s^{\prime}}-\omega-i \omega^{\prime}} \\
\times\left[\frac{1}{\epsilon\left(q, i \omega^{\prime}\right)}-1\right] \\
\sum_{s}^{\mathrm{pole}}(\mathbf{k}, \omega)=\sum_{s^{\prime} \mathbf{q}}\left[\theta\left(\omega-\xi_{\mathbf{k}+\mathbf{q}, s^{\prime}}\right)-\theta\left(-\xi_{\mathbf{k}+\mathbf{q}, s^{\prime}}\right)\right] \\
\times V_{c}(\mathbf{q}) F_{s s^{\prime}}(\mathbf{k}, \mathbf{k}+\mathbf{q})\left[\frac{1}{\epsilon\left(q, \xi_{\mathbf{k}+\mathbf{q}, s^{\prime}}-\omega\right)}-1\right] .
\end{gathered}
$$

The $\Sigma^{\text {ex }}$ and $\Sigma^{\text {line }}$ are completely real, the latter because $\epsilon(q, i \omega)$ is real. Thus, $\operatorname{Im}\left[\Sigma_{s}^{\text {pole }}(\mathbf{k}, \omega)\right]$ gives the total contribution to the imaginary part of the self-energy, i.e.,

$$
\begin{gathered}
\operatorname{Im}\left[\Sigma_{s}^{\mathrm{ret}}(\boldsymbol{k}, \omega)\right]=\sum_{s^{\prime}} \int \frac{d \boldsymbol{q}}{(2 \pi)^{2}}\left[\theta\left(\omega-\xi_{\boldsymbol{k}+\boldsymbol{q}, s^{\prime}}\right)-\theta\left(-\xi_{\boldsymbol{k}+\boldsymbol{q}, s^{\prime}}\right)\right] \\
\times V_{c}(q) \operatorname{Im}\left[\frac{1}{\varepsilon\left(q, \xi_{\boldsymbol{k}+\boldsymbol{q}, s^{\prime}}-\omega\right)}\right] F_{s s^{\prime}}(\boldsymbol{k}, \boldsymbol{k}+\boldsymbol{q}) .
\end{gathered}
$$

The inverse quasiparticle lifetime (or, equivalently, the scattering rate) $\Gamma_{s}(\boldsymbol{k})$ of state $|s \boldsymbol{k}\rangle$ is obtained by setting the frequency in imaginary part of the self-energy to the on-shell (bare quasiparticle) energy $\xi_{s k}$, i.e.,

$$
\Gamma_{s}(\boldsymbol{k})=2 \operatorname{Im}\left[\Sigma_{s}^{\mathrm{ret}}\left(\boldsymbol{k}, \xi_{\boldsymbol{k} s}\right)\right]
$$

(The factor 2 comes from the squaring of the wavefunction to obtain the occupation probability.) The $G_{0} W$ self-energy approximation used here is equivalent to the Born approximation for the scattering rate. Note that the integrand of Eq. (5) is non-zero only when $\operatorname{Im}[\epsilon] \neq 0$ or $\operatorname{Re}[\epsilon]=0$. These correspond to scattering off singleparticle excitations and plasmons, respectively.

\section{QUASI-PARTICLE SCATTERING RATE}

The self-energies and quasiparticle lifetimes of graphene and conventional parabolic-band semiconductors differ considerably. These differences can be explained with the help of Fig. 1, which shows the singleparticle excitation (SPE) and injected-electron energy loss (IEEL) continua and the plasmon dispersion for a direct gapless 2D parabolic band semiconductor and for graphene. The intersections of the IEEL continua with the SPE continua and the plasmon dispersion lines indicate allowed decay processes via $e-e$ interactions. In both doped parabolic-band semiconductors and graphene, an injected electron will decay via single-particle intraband excitations of electrons in the conduction band. In parabolic band semiconductors, an electron injected with sufficient kinetic energy can also decay via plasmon emissions and interband SPE (also known as "impact ionization"). On the other hand, as shown in Fig. 1(b), electrons injected into doped graphene cannot decay via plasmon emission, and the region in $q-\omega$ where decay via interband SPE is allowed (along the straight line segment between $\omega=v_{0} k_{F}$ and $v_{0}\left(k-k_{F}\right)$, for $\left.k>2 k_{F}\right)$ is of measure zero. In fact, within the Born approximation the quasiparticle lifetime of graphene due to $e-e$ interactions at $T=0$ is infinite. (Multiparticle excitations, which are excluded in the approximations used here, will give a quasiparticle a finite lifetime ${ }^{11}$, but this is a relatively small effect in graphene.)

In doped graphene, the only independent parameters relevant for Born appxoimation quasiparticle scattering rates at $T=0$ are the Fermi energy relative to the Dirac 

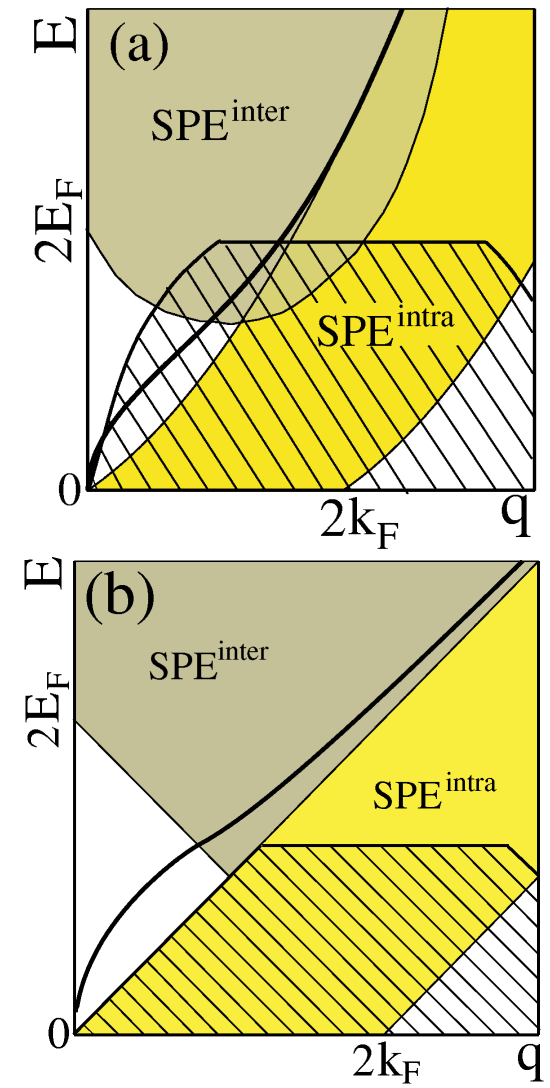

FIG. 1: (Color online) The single-particle excitations (intraband and interband) and injected-particle energy-loss (hatched region) continua, and plasmon dispersion (thick line) for (a) gapless parabolic-band semiconductor with equal hole and electron masses and (b) graphene, at $T=0$ with Fermi energy in the the conduction band. For gapped semiconductors, the interband continua are shifted up by the energy gap.

point $E_{F}=v_{0} k_{F}$ and the dimensionless coupling constant $r_{s}=e^{2} /\left(\kappa v_{0}\right)$. The scattering rate, which has units of energy, must therefore be proportional to $E_{F}$, and must be a function only of $\epsilon / E_{F}=k / k_{F}$ and $r_{s}$. Fig. 2(a) shows the Born approximation $T=0$ quasiparticle lifetime $1 / \tau=\Gamma$ due to $e-e$ interactions as a function of energy $\xi(k)=\epsilon_{k}-E_{F}$. Since the speed of the quasiparticles close the the Dirac points is approximately a constant $v_{0} \approx 10^{8} \mathrm{~cm} / \mathrm{s}$, the inelastic mean free path $\ell$ is obtained by $\ell(\xi)=v_{0} \tau(\xi)$. In Fig. 2(b), we provide the corresponding $\ell$, which shows that at $n=10^{13} \mathrm{~cm}^{-2}$ a hot electron injected with an energy of $1 \mathrm{eV}$ above $E_{F}$ has an $\ell$ due to $e-e$ interactions that is on the order of $20 \mathrm{~nm}$. This will have implications for designing any hot electron transistor type graphene devices. In particular, because Klein tunneling $\frac{12}{2}$ in graphene creates problems in the standard gate-potential switching method in transistors, in its place could be a switch based on modulating the electron energy $\xi$ a regime where $|\partial \ell / \partial \xi|$ is large.

As with doped parabolic-band 2D semiconductors $13,14,15$, in graphene $\Gamma(k)=1 / \tau(k) \propto$ $\left(k-k_{F}\right)^{2}\left|\log \left(\left|k-k_{F}\right|\right)\right|$ for $k \approx k_{F}$ due to scatter-
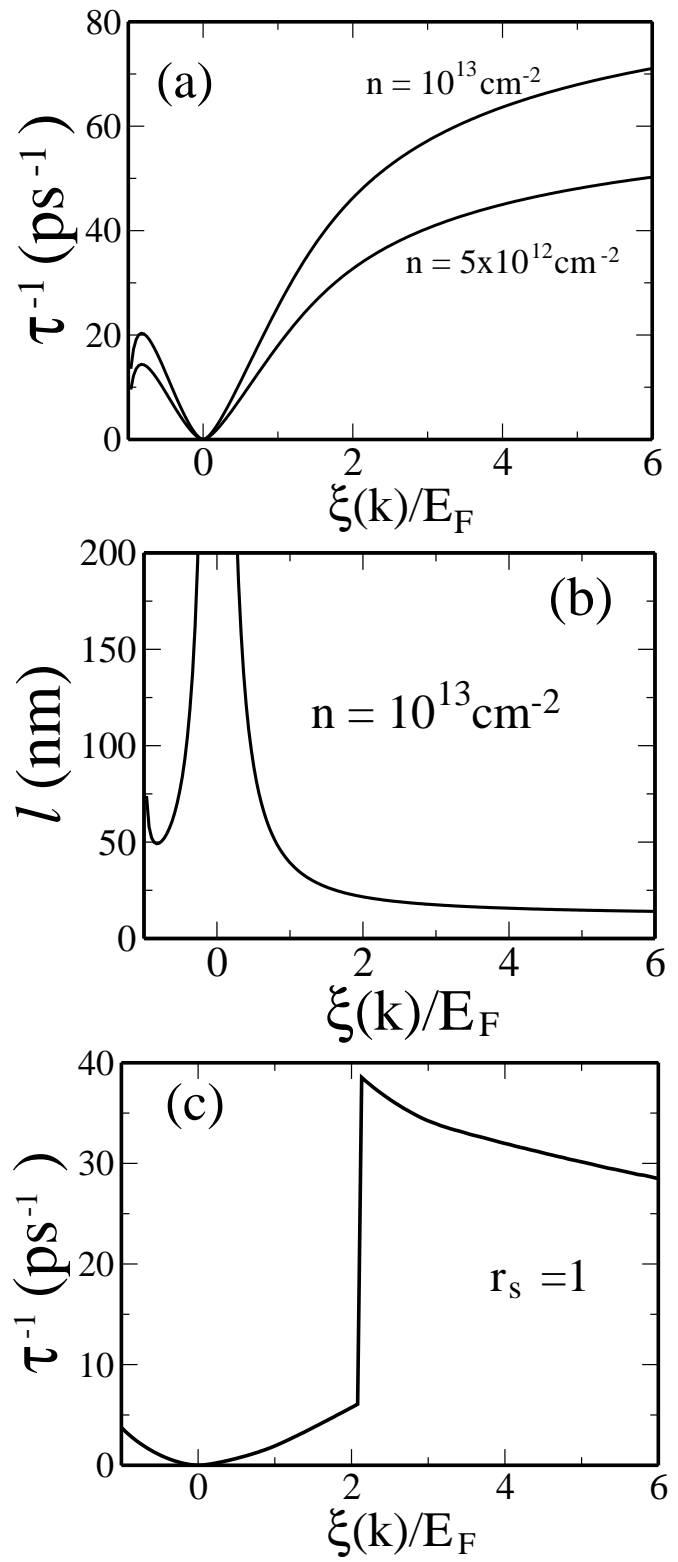

FIG. 2: (a) Inelastic quasiparticle lifetime/scattering rate $(1 / \tau=\Gamma)$ in graphene due to dynamically screened $e-e$ interactions, as a function of energy at $T=0$ for different densities, within the Born approximation. (b) The corresponding quasiparticle mean free path for $n=10^{13} \mathrm{~cm}^{-2}$ (corresponding to $E_{F} \approx 0.4 \mathrm{eV}$ ). (c) Equivalent scattering rate for a parabolic band semiconductor (without interband processes), for comparison.

ing phase-space restrictions. Further away from $k_{F}$, however, the dependence of $\Gamma$ on $k$ in graphene and in parabolic-band semiconductors are markedly and qualitatively different. To wit, in parabolic band semiconductors plasmon emission $\underline{14,16}$ and interband collision thresholds 17 cause discontinuities in the $\Gamma(k)$, as shown in Fig. 2(c), but the graphene $\Gamma(k)$ is a smooth function because both plasmon emission and interband processes are absent. 


\section{SELF ENERGY}

In order to see the effects of the plasmons and interband $\mathrm{SPE}$ in graphene in $\operatorname{Im}\left[\Sigma^{\text {ret}}\right]$, one must look off-shell, i.e., $\omega \neq \xi_{\boldsymbol{k}, s}$. The off-shell $\operatorname{Im}\left[\Sigma_{s}^{\mathrm{ret}}\right]$ is not merely of academic interest; it is needed to interpret data from ARPES. The spectra of the ARPES electrons ejected from graphene give the electronic spectral function, from which one can infer $\Sigma_{s}^{\text {ret }}(\boldsymbol{k}, \omega)^{18}$. Physically, in the $G_{0} W$ approximation, the off-shell $2 \operatorname{Im}\left[\Sigma_{s}^{\text {ret }}(\mathbf{k}, \omega)\right]$ gives the Born approximation decay rate of the quasiparticle in state $\boldsymbol{k}$ if it had kinetic energy $\omega+E_{F}$ rather than $\xi_{\boldsymbol{k}}+E_{F}$.

In Fig. 3 we show $\operatorname{Im}\left[\Sigma_{+}^{\text {ret }}(k, \omega)\right]$ for $k=0$ and $k=k_{F}$ as a function of $\omega$. Within the $G_{0} W$ approximation, the contributions to the off-shell $\operatorname{Im}\left[\Sigma_{s}^{\mathrm{ret}}(\boldsymbol{k}, \omega)\right]$ can be visualized as the intersection of the SPE continuum and plasmon line in Fig. 1 with the vertically displaced IEEL ${ }^{19}$. At $k=0$ there are two contributions to $\operatorname{Im}[\Sigma]$, the intraband and interband SPEs. For low energies $\left(|\omega| \lesssim E_{F}\right)$ only intraband SPE contributes to $\operatorname{Im}[\Sigma]$. Its contribution reaches a maximum around Fermi energy, then decreases gradually with increasing energy, as is the case with a parabolic-band semiconductor ${ }^{16}$ where it is the only decay channel for the quasiparticle (assuming $\omega<$ band gap energy). But in graphene there is a new decay channel of the quasiparticle, the interband SPE. Due to the phase space restrictions the interband SPE does not contribute to the self energy at low energies, but at higher energies $\left(\omega \gtrsim E_{F}\right)$ its contribution increases sharply, overwhelming the SPE ${ }^{\text {intra }}$ contribution. The $\mathrm{SPE}^{\text {inter }}$ contribution then increases almost linearly with $\omega$, with the same slope as for intrinsic graphene ${ }^{8}$. Plasmons do not contribute to $\operatorname{Im}\left[\Sigma_{+}^{\text {ret }}(\boldsymbol{k}=0, \omega)\right]$ for $\omega>0$.

At $k=k_{F}$, not only do plasmons contribute to $\operatorname{Im}[\Sigma]$, in the low-energy $\left(\omega \lesssim 2 E_{F}\right)$ regime, they actually dominate over the SPE contributions, as can be seen in Fig. 3(b). In contrast, in parabolic-band 2DEGs the plasmon and SPE contributions to $\operatorname{Im}\left[\Sigma^{\text {ret }}\left(k_{F}, \omega\right)\right]$ go as $\omega^{2}$ and $\omega^{2} \ln \omega$, respectively ${ }^{14}$, and hence both contributions are roughly equal in magnitude.

Fig. 4 shows the imaginary part of the quasiparticle self-energies for the conduction band, $\operatorname{Im}\left[\Sigma_{+}^{\text {ret }}\right]$, and the valence band, $\operatorname{Im}\left[\Sigma_{-}^{\text {ret }}\right]$, of graphene for several different wavevectors. For $k>k_{F}$, the $\operatorname{Im}\left[\Sigma_{+}^{\text {ret }}\right]$ shows a sharp peak associated with the plasmon emission threshold. In the high energy regime, the dominant contribution to $\operatorname{Im}[\Sigma]$ comes from the interband SPE, which gives rise to an $\operatorname{Im}[\Sigma]$ is linear in $\omega$ for all wavevectors. Note that within the $G_{0} W$ approximation, for a given $r_{s}$ in graphene, the $\Sigma, \omega$ and $k$ scale with $E_{F}, E_{F}$ and $k_{F}$, respectively; i.e., for fixed $r_{s}$, the function $\tilde{\Sigma}(\tilde{k}, \tilde{\omega})$ is universal, where $\tilde{\Sigma}=\Sigma / E_{F}, \tilde{k}=k / k_{F}$ and $\tilde{\omega}=\omega / E_{F}$.

Finally, Fig. 5 shows the calculated $\operatorname{Im}\left[\Sigma_{+}^{\text {ret }}\right]$ at fixed $k=k_{F}$ as a function of energy $\omega$ for various densities. No fitting parameters were used in this calculation. These results compare favorably with the recent data from ARPES experiments by Bostwick, et al $\stackrel{3}{\underline{3}}$ from
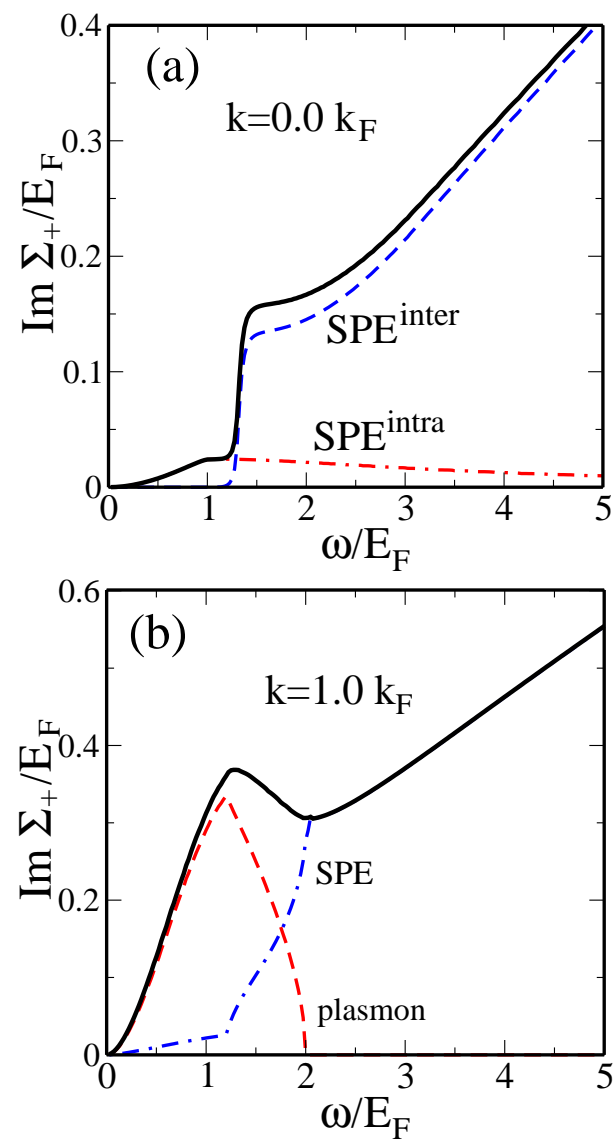

FIG. 3: (Color online) The imaginary part the retarded selfenergy at $T=0$ (thick line) for (a) $k=0$ and (b) $k=k_{F}$ as a function of energy. The dot-dashed and dashed lines in (a) are the $\mathrm{SPE}^{\text {intra }}$ and $\mathrm{SPE}^{\text {inter }}$ contributions, respectively. The dot-dashed and the dashed lines in (b) are the total SPE (intraband and interband SPE) and the plasmon contributions, respectively.

which they extract $\operatorname{Im}\left[\Sigma^{\mathrm{ret}}\left(k_{F}, \omega\right)\right]$ for different densities. (The overall scale is different because we have assumed a $\mathrm{SiO}_{2}$ substrate used by some other groups, whereas Ref. 3's samples were on $\mathrm{SiC}$, which has a different $\kappa$.) Bostwick, et al. invoked plasmons, SPE and phonon effects in interpreting their data. We find that including just the plasmon and SPE effects, we get reasonable agreement with their data, except for features near $0.2 \mathrm{eV}$ which probably can be explained by calculations that include electron-phonon interactions.

Before concluding, we discuss some of the approximations that lead to Eq. (1). We ignore on-site (Hubbard) $e-e$ interactions because, at zero magnetic field, this interaction is irrelevant in the renormalization group sense $^{20}$. Our calculation does include both intra- and inter-band scattering processes, since our expression for $\Sigma_{s}\left(\mathbf{k}, i \omega_{n}\right)$, Eq. (1), contains a pseudospin sum $s^{\prime}= \pm 1$. Finally, there is an issue about the coupling of the bands due to interactions; i.e., because of non-diagonal terms $\Sigma_{s s^{\prime}}$ with $s \neq s^{\prime}$, the elementary excitations are a super- 

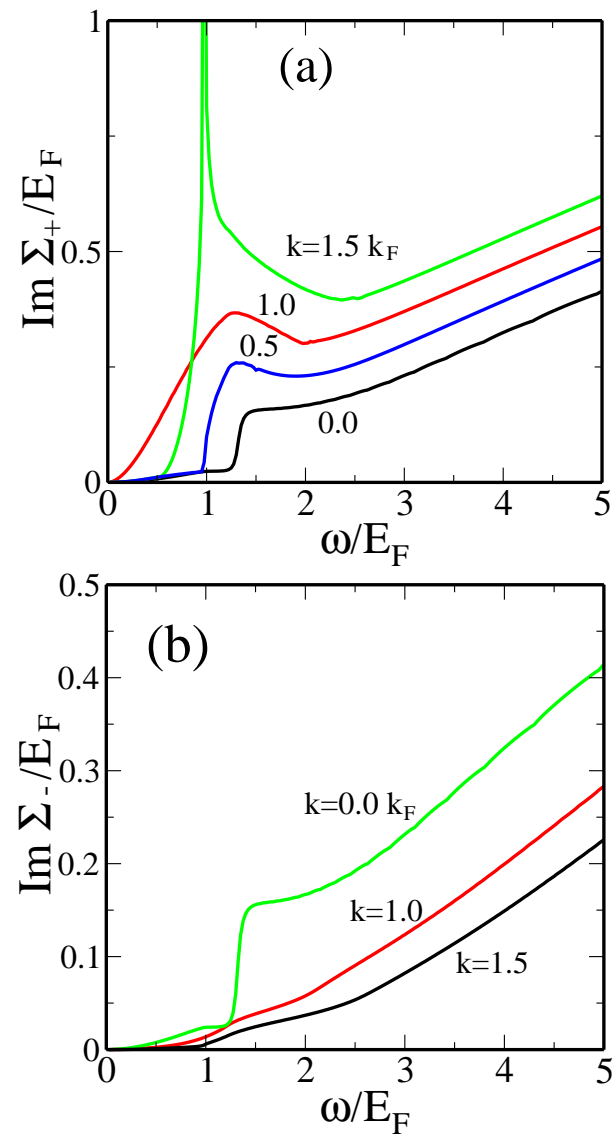

FIG. 4: (Color online) The imaginary part of the self energy as a function of energy for wavevectors $k=0,0.5,1.0$, $1.5 k_{F}$ for (a) the conduction band and (b) the valence band of electron-doped graphene at $T=0$.

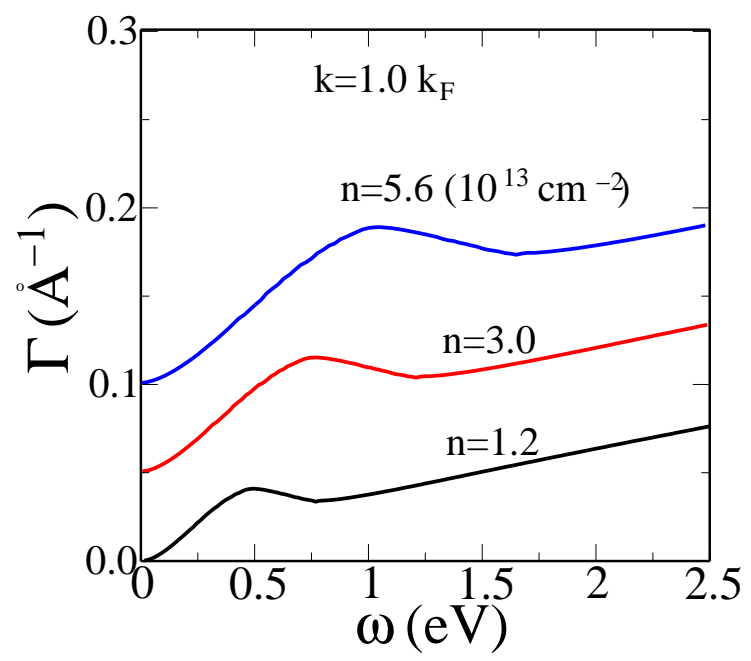

FIG. 5: (Color online) Scattering rate as a function of energy for different densities, $n=1.2,3.0,5.6 \times 10^{13} \mathrm{~cm}^{-2}$ (for clarity, successive lines are shifted upward by $0.05 \AA^{-1}$ ), in the form of Fig. 3 of Bostwick et al. $\stackrel{3}{\text {. }}$. position of states from the conduction and the valence band. Dyson's equation in this case is $\mathbf{G}^{-1}=\mathbf{G}_{0}^{-1}-\boldsymbol{\Sigma}$, where the quantities are $2 \times 2$ matrices, which, when written out in full, is

$$
\begin{aligned}
& \mathbf{G}^{-1}(\mathbf{k}, \omega)= \\
& \left(\begin{array}{cc}
\omega-\xi_{+}(k)-\Sigma_{++}(\mathbf{k}, \omega) & -\Sigma_{+-}(\mathbf{k}, \omega) \\
-\Sigma_{-+}(\mathbf{k}, \omega) & \omega-\xi_{-}(k)-\Sigma_{--}(\mathbf{k}, \omega)
\end{array}\right)
\end{aligned}
$$

The excitation energies are given by the poles of $\mathbf{G}$, or equivalently, the zeroes of the determinant of $\mathbf{G}^{-1}$. Our calculation is equivalent to ignoring the off-diagonal terms $\Sigma_{+-} \Sigma_{-+}$in the determinant of $\mathbf{G}^{-1}$. The offdiagonal terms $\Sigma_{ \pm \mp}$ are each of order $r_{s}$, and therefore the product $\Sigma_{+_{-}} \Sigma_{-+} \sim r_{s}^{2}$ which can be ignored in the weak-coupling regime. We also ignore intervalley scattering because the Born approximation Coulomb scattering rate in $2 \mathrm{D}$ is $\propto q^{-2}$, implying that small- $q$ intravalley scattering processes dominate over the large- $q$ intervalley ones.

\section{CONCLUSION}

To conclude, we have calculated the electron-electron interaction induced hot electron inelastic scattering in graphene, finding a number of intriguing and significant differences with the corresponding $2 \mathrm{D}$ parabolic dispersion systems. Our infinite ring-diagram $G_{0} W$ appracomation should be an excellent quantitative approximation for graphene since graphene is a low- $r_{s}$ (i.e., weakcoupling) system. Our $T=0$ results should remain for $T \ll T_{F}$, which is typically the case in experiments. We obtain good agreement with recent ARPES data without invoking any phonon effects. ARPES data has recently created a controversy with respect to the role of plasmons 3.4 . Our detailed calculation using a realistic model generally agrees with the interpretation of the data in Ref. 3. The calculated inelastic scattering length $\ell$ as a function of energy $\xi$ is by itself is of interest in the context of ballistic hot electron transistor applications of graphene, where the performance limitation is inelastic scattering. Furthermore, the Klein tunneling effect in graphene makes it difficult to switch transistors by a gate-voltage induced depletion (as in a conventional MOSFET). In its place, one could imagine a graphenebased transistor in which switching is achieved by modulation of the injection carrier energy in the regime of $\xi$ where $|d \ell(\xi) / d \xi|$ is large.

This work is supported by US-ONR. 
${ }^{1}$ K. S. Novoselov, A. K. Geim, S. V. Morozov, D. Jiang, Y. Zhang, S. V. Dubonos, I. V. Grigorieva, and A. A. Firsov, Science 306, 666 (2004); Nature 438, 197 (2005).

2 See e.g., C. Berger, Z. Song, T. Li, X. Li, A. Y. Ogbazghi, R. Feng, Z. Dai, A. N. Marchenkov, E. H. Conrad, P. N. First, and W. A. de Heer, J. Phys. Chem. B 108, 19912 (2004); Y. Zhang, Y.-W. Tan, H. L. Stormer, and P. Kim, Nature 438, 201 (2005); J. S. Bunch, Y. Yaish, M. Brink, K. Bolotin, and P. L. McEuen, Nano Lett. 5, 2887 (2005); A. K. Geim and K. S. Novoselov, Nature Materials 6, 183 (2007), and references therein.

3 A. Bostwick, T. Ohta, T. Seyller, K. Horn, and E. Rotenberg, Nature Physics 3, 36 (2007).

${ }^{4}$ S. Y. Zhou, G.-H. Gweon, J. Graf, A. Fedorov, C. Spataru, R. Diehl, Y. Kopelevich, D.-H. Lee, S. G. Louie, and A. Lanzara, Nature Physics 2, 595 (2006).

5 See, e.g., G.D. Mahan, Many Particle Physics, 3rd ed. (Kluwer/Plenum, New York, 2000).

${ }^{6}$ E. H. Hwang and S. Das Sarma, Phys. Rev. B 75, 205418 (2007).

7 K. W. K. Shung, Phys. Rev. B 34, 979 (1986).

8 S. Das Sarma, E. H. Hwang, and W.-K. Tse, Phys. Rev. B 75, 121406(R) (2007).

9 E. H. Hwang, Ben Yu-Kuang Hu, and S. Das Sarma, arXiv:cond-mat/0703499 Yafis Barlas, T. Pereg-Barnea, Marco Polini, Reza Asgari, and A. H. MacDonald, Phys. Rev. Lett. 98, 236601 (2007).

10 John J. Quinn and Richard A. Ferrell, Phys. Rev. 112, 812 (1958).
11 J. González, F. Guinea, and M. A. H. Vozmediano, Phys. Rev. Lett. 77, 3589 (1996).

12 O. Klein, Z. Physik 53, 157 (1929); M. I. Katsnelson, K. S. Novoselov and A. K. Geim, Nature Physics 2, 620 (2006); J. Milton Pereira, Jr., V. Mlinar, F. M. Peeters, and P. Vasilopoulos, Phys. Rev. B 74, 045424 (2006); V. V. Cheianov and V. I. Falko, Phys. Rev. B 74, 041403(R) (2006).

13 A. V. Chaplik, Zh. Eksp. Teor. Fiz. 60, 1845 (1971) [Sov. Phys. JETP 33, 997 (1971)]; C. Hodges, H. Smith, and J. W. Wilkins, Phys. Rev. B 4, 302 (1971).

14 G. F. Giuliani and J. J. Quinn, Phys. Rev. B 26, 4421 (1982)

15 Lian Zheng and S. Das Sarma, Phys. Rev. B 53, 9964 (1996); T. Jungwirth and A. H. MacDonald, Phys. Rev. B 53, 7403 (1996)

16 R. Jalabert and S. Das Sarma, Phys. Rev. B 40, 9723 (1989).

17 L. V. Keldysh, zh. Eksp. Teor. Fiz. 48, 1692 (1965); [Sov. Phys. - JETP 21, 1135 (1965)].

18 For a review of ARPES, see e.g., A. Damascelli, Z. Hussain and Z.-X. Shen, Rev. Mod. Phys. 75, 473 (2003).

19 More accurately, the contributions come from the regions in the $E-q$ plane between $E=0$ and $E=\omega$ in which the $\mathrm{SPE}$ and plasmon lines intersect with the energy loss and gain spectrum that is shifted along the $E$-axis by $\omega-\xi_{k}$.

20 J. González, F. Guinea, and M. A. H. Vozmediano, Phys. Rev. B 63, 134421 (2001). 